\title{
The Associations of Gender, Menopause, Age, and Asthma with REM-Predominant Obstructive Sleep Apnea: A Prospective Observational Study
}

This article was published in the following Dove Press journal: Nature and Science of Sleep

\author{
Rakan A Bahammam (D) \\ Khalid M Al-Qahtani' \\ Salih A Aleissi (D)' \\ Awad H Olaish (1D) \\ Aljohara S Almeneessier $\mathbb{D}^{1,2}$ \\ Ahmed S Bahammam (D) \\ 'Department of Medicine, College of \\ Medicine, The University Sleep Disorders \\ Center, King Saud University, Riyadh, \\ Saudi Arabia; ${ }^{2}$ Family Medicine, College \\ of Medicine, King Saud University, Riyadh, \\ Saudi Arabia
}

Purpose: The study sought to assess demographics, clinical features, comorbidities, and polysomnographic features of a large cohort of clinic-based patients with rapid eye movementpredominant obstructive sleep apnea (REM-predominant-OSA) in both genders, while assessing the relationship between REM-predominant OSA in one hand and menopausal status and age on the other.

Methods: This prospective observational study was conducted between January 2003 and December 2017. REM-predominant OSA diagnostic criteria included an AHI of $\geq 5 / \mathrm{h}$, with REM-AHI/non-REM-AHI of $>2$, a non-REM-AHI of $<15 / \mathrm{h}$, and a minimum of $15 \mathrm{~min}$ of REM sleep. Patients who had an AHI $>5$ events/h and did not meet the criteria for REMpredominant OSA were included in the non-stage-specific OSA group (NSS).

Results: The study consisted of 1346 men and 823 women (total=2169). REM-predominant OSA was diagnosed in $17 \%(\mathrm{n}=369)$. The prevalence of REM-predominant OSA in women was $25 \%$ compared with $12 \%$ in men. Several independent associations of REMpredominant OSA were identified in the whole group, including age (OR: 0.97 [0.95-0.98], $\mathrm{p}<0.01$ ), female sex (OR: 6.95 [4.86-9.93], $\mathrm{p}>0.01$ ), REM sleep duration ( $\min$ ) (OR: 1.02 [1.02-1.03], <0.01), and time with $\mathrm{SpO} 2<90 \%$ (mins) (OR: 0.97 [0.95-0.99], < 0.01), hypertension (OR:0.67 [0.45-0.99], 0.04) and asthma (OR: 2.19 [1.56-3.07], < 0.01). The prevalence of REM-predominant OSA in premenopausal and postmenopausal women was $35 \%$ and $18.6 \%(\mathrm{p}<0.01)$, respectively. Among women, age was an independent correlate (OR: 0.97 [0.94-0.99], $\mathrm{p}=0.03$; however, menopausal status was not.

Conclusion: REM-predominant OSA is prevalent among clinic-based patients with OSA. A younger age and female sex were independent correlates of REM-predominant OSA. Among women, a younger age but not menopausal status was a correlate of REMpredominant OSA. Asthma was independently associated with REM-predominant OSA.

Keywords: female sex, phenotype, rapid eye movement sleep, hypertension, menopause, apnea-hypopnea index

\section{Introduction}

Obstructive sleep apnea (OSA) is a common sleep disorder that occurs during sleep and causes repetitive cessation of the upper airway and intermittent hypoxemia. The overall population-prevalence of OSA ranges from $9 \%$ to $38 \%$ and is higher among men. ${ }^{1}$ It is estimated that approximately 1 billion middle-aged adults worldwide could have OSA, and the estimated number of middle-aged adults with moderate to severe OSA, for which treatment is generally recommended, is nearly 425 million. ${ }^{2}$
Correspondence: Ahmed S Bahammam Department of Medicine, College of Medicine, The University Sleep Disorders Center, King Saud University, Box 225503, Riyadh I I324, Saudi Arabia

Tel +966- I I-467-9495

Fax +966-II-467-9I79

Email ashammam2@gmail.com 
Upper airway narrowing can occur during rapid eye movement (REM) and non-REM (NREM) sleep. In REM sleep, pharyngeal muscle activity decreases and significantly increases the inclination for upper airway collapse due to the removal of excitatory noradrenergic and serotonergic inputs to upper airway motor neurons. ${ }^{3}$ Therefore, REM sleep in some patients with OSA is characteristically accompanied by an increased incidence of obstructive events, that are frequently prolonged and followed with severe oxygen desaturation. ${ }^{4}$ REM-related OSA is used loosely by sleep clinicians when polysomnography (PSG) shows obstructive sleep-disordered breathing events that occur predominantly or exclusively in REM sleep. ${ }^{5}$ A common criterion used for diagnosis is the ratio between the apnea-hypopnea index (AHI) during REM and NREM sleep (the AHI-REM/AHI-NREM ratio), where an AHI-REM/AHI-NREM ratio $\geq 2$ indicates a preponderance of respiratory events during REM sleep, or REM-related OSA. ${ }^{5}$ However, this definition is not accurate if REM sleep duration and NREM-AHI are not considered. Therefore, we need to use criteria that account for REM sleep duration and NREM-AHI. ${ }^{5}$

Blood pressure increases following obstructive events during REM and NREM sleep. ${ }^{6}$ However, REM sleep has been shown to be accompanied by enhanced sympathetic activity and instability of the cardiovascular system; ${ }^{6}$ therefore, theoretically, REM-related OSA may be related to more cardiovascular harmful events than NREM OSA. Recently, findings have pointed to an association between REM-related OSA and increased cardiovascular risk. A cross-sectional and longitudinal association between REM-related OSA and hypertension has been shown. ${ }^{7}$

A few studies have compared REM and NREM-OSA; however, previous studies either had a small sample size or did not apply strict criteria to define REM-related OSA. Additionally, racial differences in the prevalence of REMrelated OSA have been reported, ${ }^{8}$ and no study has reported the prevalence of REM-related OSA in Saudi Arabs. Moreover, the relationship between menopausal status (using a precise definition) and REM-related OSA has not been well-explored. It has been shown that REM-related OSA is more common in women than men; however, it remains unclear whether REM-related OSA in women is related to age or premenopausal status. ${ }^{9}$ As REM-related OSA has been commonly reported in young men and women, this may basically indicate that REM-related OSA is related to a stiffer and younger airway, which is less likely to collapse during NREM sleep, rather than to menopausal status and female hormones. ${ }^{9}$ We hypothesized that REMpredominant OSA (REM-predominant OSA) in women is associated with a younger age rather than with menopausal status. Therefore, this study was designed to assess demographic characteristics, clinical features, comorbidities, and polysomnographic features of a large cohort of REMpredominant-OSA patients and compare these to non-stagespecific (NSS) OSA patients. Additionally, the study sought to assess the relation between REM-predominant OSA on one hand, and gender, age, and menopausal status on the other hand. As we used strict criteria to define REM-related OSA, we will call this REM-predominant OSA in this manuscript. Additionally, as obstructive events occur in both REM and NREM sleep, we will call OSA patients who do not meet the REM-predominant OSA diagnostic criteria, non-stagespecific OSA (NSS-OSA) patients.

\section{Methods}

In this prospective observational study, the data generated formed part of a project to assess REM-predominant OSA. $^{5,6}$ In the period between January 2003 and December 2017, all patients ( $\geq 18$-years-old) who consulted the University Sleep Disorders Centre (USDC) at King Saud University and were diagnosed with OSA based on nocturnal PSG were included. Diagnostic daytime sleep studies were excluded because the timing of REM sleep could be coupled to the circadian rhythm. ${ }^{10}$ Other exclusion criteria included sleeping less than 250 min and spending less than 15 min in REM sleep, as well as having received any previous surgical procedures for the treatment of snoring or OSA. In addition, patients with congestive heart failure, chronic neurological, muscular, or chronic obstructive pulmonary disease (COPD), wall and thoracic vertebral column deformities, daytime hypercapnia, and patients on sedative drugs were excluded. None of the included patients had been on positive airway pressure therapy or were using oral appliances at the time of recruitment.

All patients were clinically evaluated by a sleep medicine specialist to obtain a detailed medical history and physical examination. The information about comorbidities was acquired from patients' medical histories and medical records. Postmenopausal status was defined when a patient was experiencing 12 consecutive months without menstruation. ${ }^{11,12}$ Subjective sleepiness was measured via the Epworth sleepiness scale (ESS). ${ }^{13}$

Figure 1 presents the study population flowchart.

The study was approved by the Institutional Review Board of the Health Sciences Colleges Research on 
Human Subjects at King Saud University (E-19-4169). All participants signed a written informed consent form.

\section{Polysomnography (Diagnostic Sleep Study)}

All patients underwent standard PSG in accordance with the American Academy of Sleep Medicine (AASM) recommendations, ${ }^{14}$ with six leads for electroencephalography, electrooculography, electromyography for the chin and legs, electrocardiography, oxygen saturation (SPO2), thoracoabdominal movements, and airflow (thermistor and nasal pressure), using Alice ${ }^{\circledR}$ diagnostic equipment (Philips, Respironics Inc., Murrysville, PA, USA). Raw data were scored manually according to the AASM scoring criteria. ${ }^{14}$ Hypopneas scoring criteria included a decrement in nasal pressure signal by $\geq 50 \%$ for at least $\geq 10$ seconds, followed by $a \geq 3 \%$ desaturation from the pre-event baseline or arousal. The desaturation index was computed as the number of desaturations (drop in oxygen saturation of $\geq 3 \%$ ) divided by total sleep time expressed in hours. Obstructive apneas and hypopneas were calculated

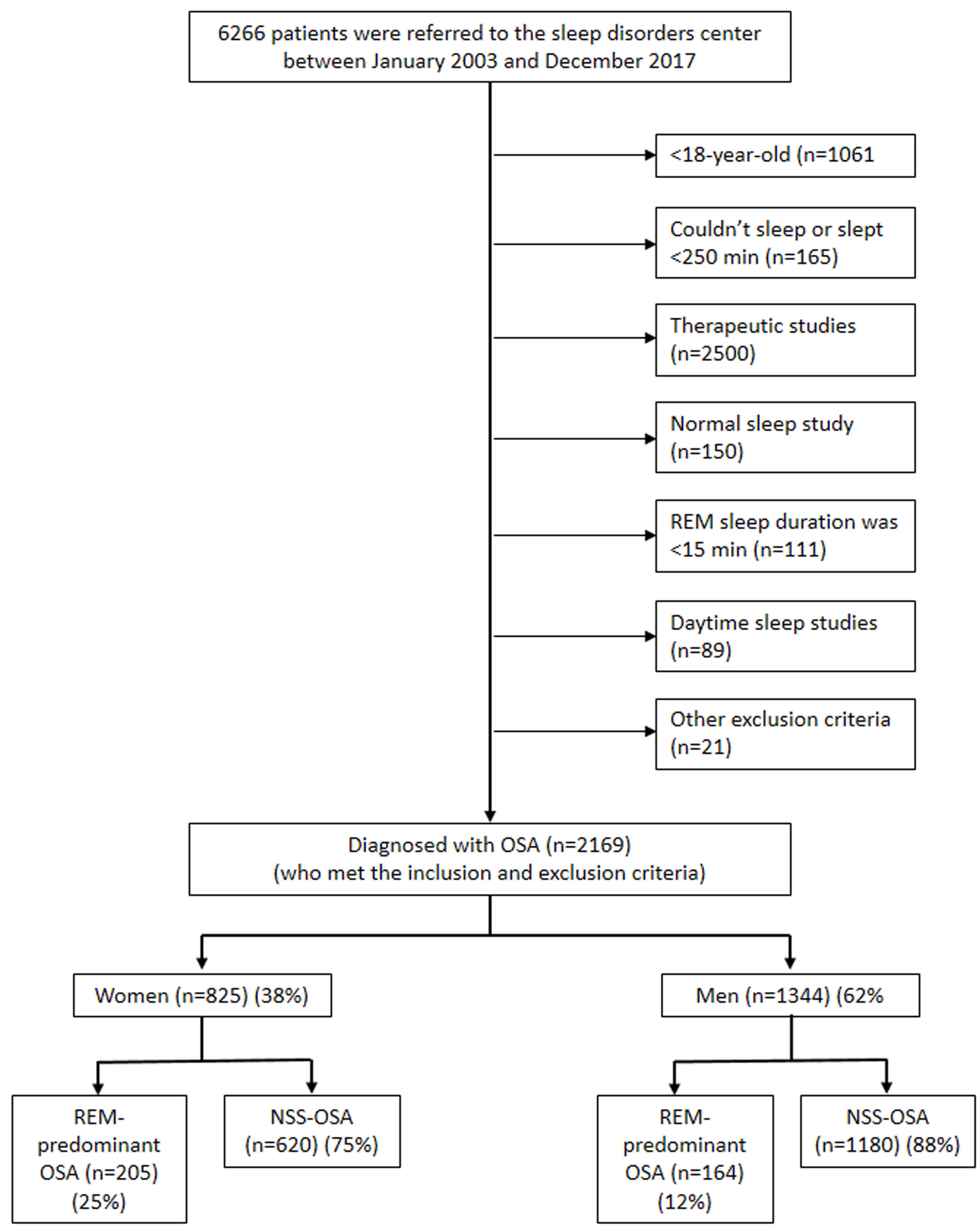

Figure I Study population flow chart.

Abbreviations: OSA, obstructive sleep apnea; NSS, non-stage-specific; REM, rapid eye movement sleep. 
during REM and NREM sleep and expressed as REMAHI and NREM-AHI.

REM-predominant OSA was defined as an obstructive AHI of $\geq 5$, with a REM-AHI that was two times the NREM-AHI (REM-AHI/NREM-AHI of more than 2), an NREM-AHI of less than 15 events/h, and a minimum of 15 min of REM sleep. ${ }^{5}$ Patients with an obstructive AHI of more than 5 events/h but did not fulfill the criteria for REM-predominant OSA were considered as having a nonstage-specific OSA group (NSS).

\section{Statistical Analysis}

Data were analyzed using SPSS statistical software (version 24; Chicago, IL, USA). Mean and standard deviation (SD) were used to describe quantitative variables. For categorical variables, frequencies and percentages were used. For the two groups comparison, a Chi-square test was used for categorical variables and a Student's $t$-test was used for normally distributed data. A Mann Whitney $U$-test was used for non-normally distributed data, based on the type of study and outcome variables.

To identify the independent correlates of REMpredominant OSA, a univariable logistic regression model was used initially where one explanatory factor was examined at a time. Next, explanatory factors with p-values less than 0.25 were entered into a multiple logistic regression model as per Hosmer-Lemeshow recommendations to identify the independent associations and correlates.

A p-value of $<0.05$ and a $95 \%$ confidence interval were used to report the statistical significance and precision of the results.

\section{Results}

The study comprised 1346 men and 823 women (total=2169). REM-predominant OSA was diagnosed in $17 \%(\mathrm{n}=369)$, and NSS-OSA was diagnosed in $83 \%$ $(\mathrm{n}=1800)$. The mean age and BMI for women were $51.7 \pm$ 12.9 years and $40.1 \pm 11.9 \mathrm{~kg} / \mathrm{m}^{2}$, respectively, and $43.7 \pm$ 13.8 years and $33.6 \pm 8.2 \mathrm{~kg} / \mathrm{m}^{2}$ for men, respectively. The prevalence of REM-predominant OSA in women was $25 \%$ compared with $12 \%$ in men.

Table 1 presents the demographic characteristics and general information of the study groups. The mean age of patients with REM-predominant OSA was $43 \pm 14$ years, compared with $47.5 \pm 13.9$ years for those with NSS-OSA $(\mathrm{P}=<0.00)$. The percentage of women in the REMpredominant OSA group was significantly higher than that in NSS-OSA group $(55 \%$ vs. $34.4 \%, \mathrm{p}<0.01)$. However, there was no statistically significant difference between the REM-predominant OSA and NSS-OSA groups in terms of BMI. The mean ESS score was significantly lower in the REM-predominant OSA group compared to the NSS-OSA group (8.6 65.6 vs. NSS-OSA group $9.8 \pm 6.4 ;<0.01)$.

In the REM-predominant OSA group, OSA was classified as mild OSA in $253(68.5 \%)$ of 368 patients, moderate in $115(31.2 \%)$, and only one severe OSA case was identified. On the other hand, in the NSS-OSA group, mild OSA was found in $299(16.6 \%)$, moderate OSA in 330 (18.3\%), and severe OSA in 1171 (65.1\%).

Women with REM-predominant OSA were significantly younger and tended to have a lower BMI compared to women with NSS-OSA $(47.2 \pm 13.2$ years vs. $53.1 \pm$ 12.5 years, $\mathrm{p}<0.01$, and $38.6 \pm 8.8 \mathrm{~kg} / \mathrm{m}^{2}$ vs. $40.6 \pm$ $12.7 \mathrm{~kg} / \mathrm{m}^{2}, \mathrm{p}=0.06$, respectively). For men, patients with REM-predominant OSA were significantly younger (37.8 \pm 13.4 years vs. $44.6 \pm 13.7$ years, $p<0.01$, respectively), but no difference in BMI was detected $\left(33.6 \pm 10 \mathrm{~kg} / \mathrm{m}^{2}\right.$ vs $33.6 \pm 8 \mathrm{~kg} / \mathrm{m}^{2}$, respectively).

\section{Presenting Symptoms and Comorbidities}

Table 2 shows the presenting symptoms and comorbidities in the studied groups. Nocturnal chest pain, awakening with a headache, and nocturnal awakening with palpitation were significantly more prevalent in the REMpredominant OSA group. However, snoring and witnessed apnea were more prevalent in the NSS-OSA group. Regarding comorbidities, insomnia, hypothyroidism, and bronchial asthma were more common among the REMpredominant OSA group, while hypertension and ischemic heart diseases were common among the NSS-OSA group.

\section{Polysomnographic Findings}

Table 3 shows the PSG parameters in both study groups. Sleep efficiency (total sleep time/time in bed) was also higher in REM-predominant OSA (84 \pm 95.2$)$ than NSSOSA $(69.6 \pm 20.1)$. Stage N3, stage REM, the lowest recorded $\mathrm{SpO} 2$, and the mean nocturnal $\mathrm{SpO} 2(\%)$ were higher in REM-predominant. The AHI was significantly lower in patients with REM-predominant OSA compared with the non-stage-specific OSA $(12.2 \pm 5.8$ vs. $53.1 \pm$ $34.5, \mathrm{p}<0.01)$, as well as the time spent with $\mathrm{SpO} 2<90 \%$ $(2.9 \pm 10.6$ min vs. $10.4 \pm 20.8 \mathrm{~min}$, respectively, $\mathrm{p}<0.01)$.

\section{Correlates of REM-Predominant OSA}

Table 4 presents the independent associations of REMpredominant OSA according to the univariable and 
Table I Demographics and General Information of Patients with REM-Predominant OSA and NSS-OSA

\begin{tabular}{|c|c|c|c|c|}
\hline Variables* & Total $n=2169$ & $\begin{array}{l}\text { REM-Predominant } \\
\text { OSA n=369 (17\%) }\end{array}$ & NSS-OSA $n=1800$ (83\%) & p-value \\
\hline Age (Year) & $46.7 \pm 14$ & $43 \pm 14.1$ & $47.5 \pm 13.9$ & $<0.01$ \\
\hline Sex (Female) & $823(37.9)$ & $203(55)$ & $620(34.4)$ & $<0.01$ \\
\hline Body Mass Index $\left(\mathrm{kg} / \mathrm{m}^{2}\right)$ & $36 \pm 10.2$ & $36.3 \pm 9.7$ & $35.9 \pm 10.4$ & 0.30 \\
\hline Neck circumference (inches) & $15.5 \pm 1.6$ & $14.9 \pm 1.5$ & $15.6 \pm 1.6$ & $<0.01$ \\
\hline Waist circumference $(\mathrm{cm})$ & $44.5 \pm 6.8$ & $43 \pm 6.5$ & $44.7 \pm 6.9$ & $<0.01$ \\
\hline Epworth Sleepiness Scale & $9.6 \pm 6.2$ & $8.6 \pm 5.6$ & $9.8 \pm 6.4$ & $<0.01$ \\
\hline Hemoglobin level $(g / L)$ & $113.2 \pm 54.3$ & $118.6 \pm 46.7$ & $112 \pm 55.7$ & 0.56 \\
\hline Hematocrit & $42.4 \pm 19.4$ & $40.3 \pm 5$ & $42.9 \pm 21.4$ & $<0.01$ \\
\hline Fasting Blood Sugar & $6.7 \pm 3.6$ & $6.1 \pm 2.1$ & $6.8 \pm 3.9$ & 0.09 \\
\hline FEVI/FVC (\%) & $85 \pm 10.4$ & $83.7 \pm 7.8$ & $85.2 \pm 10.8$ & 0.41 \\
\hline FVC (\% predicted) & $85.1 \pm 24.4$ & $85.4 \pm 20.7$ & $85 \pm 25$ & 0.10 \\
\hline FEVI (\% predicted) & $87.3 \pm 26$ & $86.7 \pm 21.7$ & $87.4 \pm 26.6$ & 0.2 \\
\hline \multicolumn{5}{|l|}{ PSG OSA severity } \\
\hline Mild OSA & $552(25.5)$ & $253(68.5)$ & $299(16.6)$ & $<0.01$ \\
\hline Moderate OSA & $445(20.5)$ & 115 (3I.2) & $330(18.3)$ & \\
\hline Severe OSA & II 72 (54) & $\mathrm{I}(0.3)$ & $117 \mid(65.1)$ & \\
\hline
\end{tabular}

Note: *Numerical data are expressed as mean \pm standard deviation, and categorical data are expressed as $\mathrm{n}(\%)$.

Abbreviations: REM, rapid eye movement; NSS, non-stage-specific; kg/m², kilogram per squared meter; cm, centimeter; g/L, gram per liter; OSA, obstructive sleep apnea.

Table 2 Symptoms and Comorbidities in Patients with REM-Predominant OSA and NSS-OSA

\begin{tabular}{|c|c|c|c|c|}
\hline Variables* & Total $n=2169$ & $\begin{array}{l}\text { REM-Predominant } \\
\text { OSA } n=369(17 \%)\end{array}$ & NSS-OSA $n=1800(83 \%)$ & p-value \\
\hline \multicolumn{5}{|l|}{ Presenting symptoms } \\
\hline Snoring & $|43|(66)$ & $203(60.4)$ & $1228(73.5)$ & $<0.01$ \\
\hline Witnessed apnea & $1077(49.7)$ & $159(47.3)$ & $918(55.3)$ & 0.01 \\
\hline Nocturnal chest pain & $555(25.6)$ & $110(32.6)$ & $445(27)$ & 0.04 \\
\hline Waking up with gastric acidity & $683(3 \mid .5)$ & $120(36.6)$ & $563(35.1)$ & 0.62 \\
\hline Nocturnal choking & $919(42.4)$ & I $43(42.4)$ & $776(46.9)$ & 0.14 \\
\hline Awakening with headache & $952(43.9)$ & $183(54.3)$ & $769(46.5)$ & 0.01 \\
\hline Nocturnal awakening with palpitation & $633(29.2)$ & $130(38.6)$ & $503(30.4)$ & $<0.01$ \\
\hline Dry mouth on awakening & $|29|(59.5)$ & $200(60.1)$ & $1091(67.1)$ & 0.014 \\
\hline Nocturia & $1267(58.4)$ & $20 \mathrm{I}(6 \mathrm{I} .3)$ & $1066(66.8)$ & 0.06 \\
\hline \multicolumn{5}{|l|}{ Comorbidities } \\
\hline Restless Legs Syndrome & $44(2)$ & $10(3.2)$ & $34(2.1)$ & 0.26 \\
\hline Insomnia & $52(2.4)$ & $16(4.4)$ & $36(2)$ & 0.01 \\
\hline Hypertension & $837(38.6)$ & $110(30.8)$ & $727(4 \mid .9)$ & $<0.01$ \\
\hline Ischemic heart disease & $123(5.7)$ & $9(2.5)$ & $114(6.6)$ & $<0.01$ \\
\hline Diabetes mellitus & $636(29.3)$ & $103(28.9)$ & $533(30.8)$ & 0.48 \\
\hline Respiratory Failure & $31(1.4)$ & $4(1.1)$ & $27(1.6)$ & 0.53 \\
\hline Compensated heart failure & $30(1.4)$ & $2(0.6)$ & $28(1.6)$ & 0.13 \\
\hline Stroke & $12(0.6)$ & $\mathrm{I}(0.3)$ & II (0.6) & 0.7 \\
\hline Bronchial asthma & 491 (22.6) & $106(29.7)$ & $385(22.2)$ & $<0.01$ \\
\hline Allergic rhinitis & $228(10.5)$ & $33(15.8)$ & $195(17.4)$ & 0.57 \\
\hline Hypothyroidism & $233(10.7)$ & $51(14.2)$ & $182(10.4)$ & 0.03 \\
\hline Hypercholesterolemia & $629(29)$ & $109(32.6)$ & $520(32.2)$ & 0.87 \\
\hline
\end{tabular}

Note: *Data are expressed as $\mathrm{n}(\%)$.

Abbreviations: REM, rapid eye movement; NSS, non-stage-specific; OSA, obstructive sleep apnea. 
Table 3 Polysomnographic Findings in Patients REM-Predominant OSA and NSS-OSA

\begin{tabular}{|c|c|c|c|c|}
\hline Variables* & Total $n=2169$ & $\begin{array}{l}\text { REM-Predominant } \\
\text { OSA } n=369(17 \%)\end{array}$ & NSS-OSA $n=1800(83 \%)$ & p-value \\
\hline Sleep efficiency (\%) & $72.2 \pm 44.6$ & $84 \pm 95.2$ & $69.6 \pm 20.1$ & $<0.01$ \\
\hline Stage NI (\%) & $10.9 \pm 10.2$ & $7.3 \pm 8$ & $11.7 \pm 10.5$ & $<0.01$ \\
\hline Stage N2 (\%) & $67.1 \pm 14.9$ & $63.4 \pm 13.5$ & $67.9 \pm 15.1$ & $<0.01$ \\
\hline Stage N3 (\%) & $4.3 \pm 8.1$ & $5.6 \pm 7.9$ & $4.1 \pm 8.2$ & $<0.01$ \\
\hline REM sleep duration (min) & $27.3 \pm 10.1$ & $50.7 \pm 15.9$ & $23.5 \pm 8.9$ & $<0.01$ \\
\hline Stage REM (\%) & $12.3 \pm 9.8$ & $18.2 \pm 7.2$ & $11.1 \pm 9.9$ & $<0.01$ \\
\hline $\mathrm{AHI}$ (events/h) & $46.2 \pm 35.1$ & $12.2 \pm 5.8$ & $53.1 \pm 34.5$ & $<0.01$ \\
\hline AHI-NREM (events/h) & $44.4 \pm 36.7$ & $6.3 \pm 3.9$ & $52.2 \pm 35.5$ & $<0.01$ \\
\hline AHI-REM (events/h) & $48.8 \pm 34.1$ & $39.8 \pm 20$ & $51.3 \pm 36.7$ & $<0.01$ \\
\hline Central apnea index (events/h) & $1.3 \pm 5.1$ & $0.1 \pm 1$ & $\mathrm{I} .6 \pm 5.6$ & $<0.01$ \\
\hline Obstructive apnea index (events/h) & $6.6 \pm 16.6$ & $0.3 \pm 0.8$ & $8 \pm 18$ & $<0.01$ \\
\hline Mixed apnea index (events/h) & $\mathrm{I} . \mathrm{I} \pm 5.5$ & $0.01 \pm 0.1$ & $1.3 \pm 6$ & $<0.01$ \\
\hline Mean duration of the obstructive event during REM (sec) & $19.1 \pm 9.8$ & $20.1 \pm 7$ & $18.9 \pm 10.3$ & 0.02 \\
\hline Mean duration of the obstructive event during NREM (sec) & $19 \pm 5.7$ & $18 \pm 5.1$ & $19.2 \pm 5.8$ & $<0.01$ \\
\hline Desaturation Index (events/h) & $25.9 \pm 30.4$ & $7 \pm 7$ & $29.8 \pm 31.9$ & $<0.01$ \\
\hline Time with $\mathrm{SpO} 2<90 \%$ (mins) & $9.1 \pm 19.7$ & $2.9 \pm 10.6$ & $10.4 \pm 20.8$ & $<0.01$ \\
\hline Time with $\mathrm{SpO} 2<95 \%$ (min) & $44.8 \pm 155.8$ & $28.9 \pm 33$ & $48.1 \pm 170.2$ & $<0.01$ \\
\hline Lowest recorded SpO2 (\%) & $82.5 \pm 11.3$ & $86.3 \pm 7.6$ & $81.7 \pm 11.8$ & $<0.01$ \\
\hline Mean nocturnal SpO2 (\%) & $94.2 \pm 5.2$ & $95.3 \pm 4.4$ & $94 \pm 5.3$ & $<0.01$ \\
\hline Arousal Index (arousals/h) & $48.9 \pm 32$ & $19.5 \pm 9.4$ & $54.9 \pm 31.6$ & $<0.01$ \\
\hline Periodic Leg Movement Index (events/h) & $2 \pm 2.2$ & $1 \pm 1.1$ & $2.1 \pm 2.3$ & $<0.01$ \\
\hline
\end{tabular}

Note: *Numerical data are expressed as mean \pm standard deviation, and categorical data are expressed as $\mathrm{n}(\%)$.

Abbreviations: REM, rapid eye movement; NREM, non-rapid eye movement; NSS, non-stage-specific; $\mathrm{kg} / \mathrm{m}^{2}$, kilogram per squared meter; cm, centimeter; g/L, gram per liter; OSA, obstructive sleep apnea; $\mathrm{AHI}$, apnea-hypopnea index; SpO2, arterial oxygen saturation; h, hour; min, minutes.

multiple logistic regression analyses in the whole group. The multiple logistic regression model identified age (OR: 0.97 [0.95-0.98], $\mathrm{p}<0.01$ ), female sex (OR: 6.95 [4.86-9.93], $\mathrm{p}>0.01$ ), and the ESS (OR: 0.94 [0.92-0.97], $<0.01)$ as independent correlates of REM-predominant OSA, and sleep efficiency (OR: 1.04 [1.03-1.05], $<0.01$ ), REM sleep duration (min) (OR: 1.02 [1.02-1.03], $<0.01$ ), and time with $\mathrm{SpO} 2<90 \%$ (mins) (OR: 0.97 [0.95-0.99], < 0.01) as PSG parameters, and hypertension (OR:0.67 [0.45-0.99], 0.04) and bronchial asthma (OR: $2.19[1.56-3.07],<0.01)$ as comorbidities.

\section{Gender and REM-Predominant OSA}

Women were divided into a premenopausal $(n=307)$ and postmenopausal $(n=516)$ group. The prevalence of REMpredominant OSA in premenopausal and postmenopausal women was $35 \%$ and $18.6 \%(\mathrm{p}<0.01)$, respectively.

Table 5 presents a comparison between the demographic characteristics, comorbidities, and PSG findings in REMpredominant OSA and non-stage-specific OSA groups categorized by sex. Patients with REM-predominant OSA were younger and had less snoring across both genders. The ESS score in women with REM-predominant OSA was lower than that in the NSS-OSA group $(7.6 \pm 5.1$ vs.9.4 \pm 5.7 , $\mathrm{p}<0.001$, respectively). Hypertension was more prevalent in the NSS-OSA group in both women and men compared to the REM-predominant OSA group.

In addition, Table 5 presents a comparison between women and men with REM-predominant OSA. Women with REM-predominant OSA were older, heavier, and had a significantly lower ESS score. Additionally, the prevalence of hypertension, diabetes mellitus, ischemic heart disease, bronchial asthma, hypothyroidism, and hypercholesterolemia was significantly higher among women with REM-predominant OSA.

To assess the impact of age and menopausal status in women, a logistic regression analysis was carried out. Table 6 presents the univariable and multiple logistic regression analyses for the correlates of REM-predominant OSA in women. Age was an independent correlate (OR: 0.97 [0.94-0.99], $\mathrm{p}=0.03$ ); however, menopausal status was not an independent correlate in the model. Other correlates of REM-predominant OSA in women were body mass index, hypertension, hypothyroidism, the Epworth sleepiness scale, stage N1 (\%), REM sleep duration, and time with $\mathrm{SpO} 2<90 \%$. 
Table 4 The Independent Correlates of REM-Predominant OSA According to the Univariable and Multiple Logistic Regression $(n=2169)$

\begin{tabular}{|c|c|c|c|}
\hline & Variables in the Equation & P-value & OR $[95 \%$ C.I.] \\
\hline \multicolumn{4}{|l|}{ Univariate model } \\
\hline & $\begin{array}{l}\text { Age (Year) } \\
\text { Body Mass Index }\left(\mathrm{kg} / \mathrm{m}^{2}\right) \\
\text { Sex (Female) } \\
\text { Hypertension } \\
\text { Diabetes mellitus } \\
\text { Hypothyroidism } \\
\text { Epworth Sleepiness Scale } \\
\text { Postmenopausal } \\
\text { Ischemic heart disease } \\
\text { Bronchial Asthma } \\
\text { Sleep Efficiency (\%) } \\
\text { Stage NI (\%) } \\
\text { Stage N2 (\%) } \\
\text { Stage N3 (\%) } \\
\text { Stage REM (\%) } \\
\text { REM sleep duration (min) } \\
\text { Apnea Hypopnea Index (events/hr) } \\
\text { Desaturation Index (desaturations/hr) } \\
\text { Time with SpO2 <90\% (mins) } \\
\text { Lowest Recorded SpO2 (\%) } \\
\text { Mean Nocturnal SpO2 (\%) }\end{array}$ & $\begin{array}{l}<0.01 \\
0.49 \\
<0.01 \\
<0.01 \\
0.48 \\
0.04 \\
<0.01 \\
<0.01 \\
<0.01 \\
<0.01 \\
<0.01 \\
<0.01 \\
<0.01 \\
0.02 \\
<0.01 \\
<0.01 \\
<0.01 \\
<0.01 \\
<0.01 \\
<0.01 \\
<0.01\end{array}$ & $\begin{array}{l}0.98[0.97-0.99] \\
1[0.99-1.01] \\
2.33[1.86-2.92] \\
0.62[0.48-0.79] \\
0.91[0.71-1.17] \\
1.43[1.03-2] \\
0.97[0.95-0.99] \\
0.43[0.31-0.59] \\
0.37[0.18-0.73] \\
1.48[1.15-1.9] \\
1.03[1.02-1.03] \\
0.94[0.92-0.95] \\
0.98[0.97-0.99] \\
1.02[1.003-1.04] \\
1.08[1.06-1.09] \\
1.03[1.03-1.04] \\
0.86[0.84-0.88] \\
0.92[0.91-0.94] \\
0.96[0.94-0.97] \\
1.06[1.04-1.07] \\
1.16[1.11-1.22]\end{array}$ \\
\hline \multicolumn{4}{|c|}{ Multiple logistic regression model ${ }^{* *}$} \\
\hline & $\begin{array}{l}\text { Age (Year) } \\
\text { Sex (Female) } \\
\text { Hypertension } \\
\text { Epworth Sleepiness Scale } \\
\text { Bronchial asthma } \\
\text { Sleep efficiency (\%) } \\
\text { REM sleep duration (min) } \\
\text { Time with SpO2 <90\% (mins) }\end{array}$ & $\begin{array}{l}<0.01 \\
<0.01 \\
0.04 \\
<0.01 \\
<0.01 \\
<0.01 \\
<0.01 \\
<0.01\end{array}$ & $\begin{array}{l}0.97[0.95-0.98] \\
6.95[4.86-9.93] \\
0.67[0.45-0.99] \\
0.94[0.92-0.97] \\
2.19[1.56-3.07] \\
1.04[1.03-1.05] \\
1.02[1.02-1.03] \\
0.97[0.95-0.99]\end{array}$ \\
\hline
\end{tabular}

Notes: **Multicollinearity: None. Overall accuracy: $96.7 \%$, Sensitivity: $88.6 \%$, Specificity: $98.1 \%$, Area under the Curve (ROC): $99.3 \%$, Omnibus Tests of Model: p<0.00I, Hosmer-Lemeshow goodness of fit: $p=0.998$, Nagelkerke R Square: $89.5 \%$.

Abbreviations: REM, rapid eye movement; OSA, obstructive sleep apnea; SpO2, arterial oxygen saturation; $h$, hour; min, minutes.

Among men (Table 6), the following independent correlates of REM-predominant OSA were identified: age, Hypertension, bronchial asthma, sleep efficiency, stage $\mathrm{N} 1(\%)$, lowest recorded SpO2, and AHI.

\section{Discussion}

This large clinic-based study used criteria based on AHI, REM-AHI/NREM-AHI ratio, and REM sleep duration to define REM-predominant OSA. With these strict criteria, REM-predominant OSA was prevalent among patients with OSA in a sleep clinic setting occurring in $17 \%$ of the studied sample. Additionally, this is the first study to assess the relationship between female sex and menopausal status with REM-predominant OSA using a clear definition for postmenopausal status. The central finding of this study is that age rather than menopausal status was an independent correlate of REM-predominant OSA in women, thereby supporting our hypothesis.

Determining the exact prevalence of REM-predominant OSA requires a clear definition and diagnostic criteria for this disorder. However, at present, there is no consensus among experts on a definition of REM-related OSA. Previous studies have reported variable prevalences, ranging from $2.7-62 \% .^{4,7-9,15-21}$ Table 7 presents a summary of previous clinic-based and community-based studies that have reported the prevalence of REM-OSA. In an earlier 
Table 5 A Comparison Between the Demographics, Comorbidities, and PSG Findings in Patients with REM-Predominant OSA and Non-Stage-Specific OSA Groups Categorized by Sex

\begin{tabular}{|c|c|c|c|c|c|c|c|c|c|}
\hline \multirow[t]{2}{*}{ Variables* } & \multicolumn{2}{|l|}{ Women } & \multirow[t]{2}{*}{ P-value } & \multicolumn{2}{|l|}{ Men } & \multirow[t]{2}{*}{ P-value } & \multicolumn{2}{|c|}{$\begin{array}{l}\text { REM-Predominant } \\
\text { OSA }\end{array}$} & \multirow[t]{2}{*}{ P-value } \\
\hline & $\begin{array}{l}\text { REM-Predominant } \\
\text { OSA }(n=203)\end{array}$ & $\begin{array}{l}\text { NSS-OSA } \\
(n=620)\end{array}$ & & $\begin{array}{l}\text { REM- } \\
\text { Predominant } \\
\text { OSA }(n=166)\end{array}$ & $\begin{array}{l}\text { NSS-OSA } \\
(n=1180)\end{array}$ & & $\begin{array}{l}\text { Women } \\
(n=203)\end{array}$ & $\begin{array}{l}\text { Men } \\
(n=166)\end{array}$ & \\
\hline \multicolumn{10}{|c|}{ Demographics and general information } \\
\hline Age (Year) & $47.2 \pm 13.2$ & $53.1 \pm 12.5$ & $<0.01$ & $37.8 \pm 13.4$ & $44.6 \pm 13.7$ & $<0.01$ & $\begin{array}{l}47.2 \pm \\
13.2\end{array}$ & $\begin{array}{l}37.8 \pm \\
13.4\end{array}$ & $<0.01$ \\
\hline $\begin{array}{l}\text { Body Mass Index (kg/ } \\
\left.\mathrm{m}^{2}\right)\end{array}$ & $38.6 \pm 8.8$ & $40.6 \pm 12.7$ & 0.06 & $33.6 \pm 10$ & $33.6 \pm 8$ & 0.20 & $38.6 \pm 8.8$ & $\begin{array}{l}33.6 \pm \\
10\end{array}$ & $<0.01$ \\
\hline $\begin{array}{l}\text { Epworth Sleepiness } \\
\text { Scale }\end{array}$ & $7.6 \pm 5.1$ & $9.4 \pm 5.7$ & $<0.01$ & $9.8 \pm 5.9$ & $10 \pm 6.7$ & 0.94 & $7.6 \pm 5.1$ & $9.8 \pm 5.9$ & $<0.01$ \\
\hline Postmenopausal & $96(47.3)$ & $420(67.7)$ & $<0.01$ & - & - & - & $96(47.3)$ & - & - \\
\hline Snoring & $109(59.9)$ & $415(70.7)$ & 0.01 & $94(6 \mathrm{I})$ & $813(75)$ & $<0.01$ & $109(59.9)$ & $94(61)$ & 0.83 \\
\hline Witnessed apnea & $82(44.8)$ & $310(53)$ & 0.05 & $77(50.3)$ & $608(56.6)$ & 0.15 & $82(44.8)$ & $\begin{array}{l}77 \\
(50.3)\end{array}$ & 0.31 \\
\hline \multicolumn{10}{|l|}{ Comorbidities } \\
\hline Hypertension & $8 I(4 I . I)$ & $364(60.7)$ & $<0.01$ & $29(18.1)$ & $363(32)$ & $<0.01$ & $8 I(4 I .1)$ & $\begin{array}{l}29 \\
(18.1)\end{array}$ & $<0.01$ \\
\hline Ischemic heart disease & $8(4.1)$ & $54(9)$ & 0.03 & $\mathrm{I}(0.6)$ & $60(5.3)$ & 0.01 & $8(4.1)$ & $\mathrm{I}(0.6)$ & 0.04 \\
\hline Diabetes mellitus & $73(37.2)$ & $268(44.9)$ & 0.06 & $30(18.6)$ & $265(23.3)$ & 0.18 & $73(37.2)$ & $\begin{array}{l}30 \\
(18.6)\end{array}$ & $<0.01$ \\
\hline Bronchial asthma & $68(34.5)$ & $201(33.6)$ & 0.82 & $38(23.8)$ & $184(16.2)$ & 0.02 & $68(34.5)$ & $\begin{array}{l}38 \\
(23.8)\end{array}$ & 0.03 \\
\hline Hypothyroidism & 47 (23.9) & $128(2||)$. & 0.42 & $4(2.5)$ & $54(4.7)$ & 0.20 & 47 (23.9) & $4(2.5)$ & $<0.01$ \\
\hline Hypercholesterolemia & $73(39.9)$ & $241(42.7)$ & 0.50 & $36(23.8)$ & $279(26.5)$ & 0.48 & $73(39.9)$ & $\begin{array}{l}36 \\
(23.8)\end{array}$ & $<0.01$ \\
\hline \multicolumn{10}{|c|}{ Polysomnographic findings } \\
\hline Sleep Efficiency (\%) & $85.5 \pm 127.8$ & $64 \pm 20.5$ & $<0.01$ & $82.2 \pm 13.8$ & $72.6 \pm 19.3$ & $<0.01$ & $\begin{array}{l}85.5 \pm \\
127.8\end{array}$ & $\begin{array}{l}82.2 \pm \\
13.8\end{array}$ & $<0.01$ \\
\hline Stage NI (\%) & $7.8 \pm 6.9$ & $12.3 \pm 11.4$ & $<0.01$ & $6.8 \pm 9.2$ & $11.4 \pm 9.9$ & $<0.01$ & $7.8 \pm 6.9$ & $6.8 \pm 9.2$ & 0.01 \\
\hline Stage N2 (\%) & $64.2 \pm 13.5$ & $67.2 \pm 16$ & 0.01 & $62.5 \pm 13.5$ & $68.3 \pm 14.6$ & $<0.01$ & $\begin{array}{l}64.2 \pm \\
13.5\end{array}$ & $\begin{array}{l}62.5 \pm \\
13.5\end{array}$ & 0.26 \\
\hline Stage N3 (\%) & $4.7 \pm 7.4$ & $4.4 \pm 9.2$ & 0.24 & $6.7 \pm 8.4$ & $3.9 \pm 7.6$ & $<0.01$ & $4.7 \pm 7.4$ & $6.7 \pm 8.4$ & 0.03 \\
\hline Stage REM (\%) & $17.1 \pm 7$ & $10.3 \pm 9.9$ & $<0.01$ & $19.5 \pm 7.2$ & $11.6 \pm 9.8$ & $<0.01$ & $17.1 \pm 7$ & $\begin{array}{l}19.5 \pm \\
7.2\end{array}$ & $<0.01$ \\
\hline $\mathrm{AHI}$ & $12 \pm 6$ & $51.4 \pm 34.6$ & $<0.01$ & $12.4 \pm 5.5$ & $54.1 \pm 34.4$ & $<0.01$ & $12 \pm 6$ & $\begin{array}{l}12.4 \pm \\
5.5\end{array}$ & 0.27 \\
\hline $\begin{array}{l}\text { Desaturation Index } \\
\text { (desaturations/hr.) }\end{array}$ & $7.2 \pm 7.3$ & $27.7 \pm 30.3$ & $<0.01$ & $6.7 \pm 6.7$ & $30.9 \pm 32.6$ & $<0.01$ & $7.2 \pm 7.3$ & $6.7 \pm 6.7$ & 0.30 \\
\hline $\begin{array}{l}\text { Time with } \mathrm{SpO} 2<90 \% \\
(\mathrm{~min})\end{array}$ & $3.6 \pm 12.5$ & $11.3 \pm 22.1$ & $<0.01$ & $2 \pm 7.6$ & $10 \pm 20.1$ & $<0.01$ & $3.6 \pm 12.5$ & $2 \pm 7.6$ & 0.22 \\
\hline $\begin{array}{l}\text { Lowest Recorded } \\
\text { SpO2 (\%) }\end{array}$ & $85.9 \pm 7.6$ & $81.5 \pm 12.5$ & $<0.01$ & $86.8 \pm 7.7$ & $81.8 \pm 11.4$ & $<0.01$ & $85.9 \pm 7.6$ & $\begin{array}{l}86.8 \pm \\
7.7\end{array}$ & 0.14 \\
\hline $\begin{array}{l}\text { Mean Nocturnal SpO2 } \\
\text { (\%) }\end{array}$ & $95.3 \pm 5.8$ & $94.2 \pm 5.1$ & $<0.01$ & $95.3 \pm 1.6$ & $93.9 \pm 5.4$ & $<0.01$ & $95.3 \pm 5.8$ & $\begin{array}{l}95.3 \pm \\
1.6\end{array}$ & 0.01 \\
\hline
\end{tabular}

Notes: *Numerical data are expressed as mean \pm standard deviation, and categorical data are expressed as $\mathrm{n}(\%)$.

Abbreviations: REM, rapid eye movement; OSA, obstructive sleep apnea; SpO2, arterial oxygen saturation; h, hour; min, minutes; kg/m², kilogram per squared meter. 
study, O'Conner et al used a definition that comprised patients with a total AHI ranging from $5 / \mathrm{h}$ to $25 / \mathrm{h}$, an NREM-AHI $<15 / \mathrm{h}$ and REM-AHI/NREM-AHI of over 2, and a reported a prevalence of REM-OSA of $62 \%$ among women and $24 \%$ among men. ${ }^{18}$ Diagnosing patients with REM-related OSA based on the REM-AHI/NREM-AHI ratio is not accurate because it will label some patients as having REM-related OSA, even when there are coexisting significant obstructive events during NREM sleep. ${ }^{22}$ For example, if a patient has a total REM-AHI of 90 events/h and an NREM-AHI of 30 events/h, the ratio will be more than two, and he will be diagnosed with REM-related OSA, when, in fact, he also has severe NREM-OSA. Another cross-sectional study of 45 obese subjects accounted for the AHI in NREM sleep and used the following criteria to diagnose REM-related OSA: a total AHI between 5/h and 25/h, an NREM-AHI $<15 / h$, and REM-AHI/NREM AHI $>$ 2. ${ }^{19}$ Results showed that REM-OSA was diagnosed in $4 \%$ of men and $35 \%$ of women. ${ }^{19}$ However, not including a minimum duration for REM sleep is insufficient to diagnose REM-related OSA. For example, if a patient spent one minute in REM sleep and had one obstructive event during REM sleep, the REM-AHI will be 60 events/h, which can be misleading. This concept is reflected by Conwell et al's study, which assessed the impact of three different diagnostic criteria on the prevalence of REMOSA. ${ }^{8}$ The prevalence of REM-related OSA was $37 \%$ using a total $\mathrm{AHI} \geq 5$ and $\mathrm{AHI}-\mathrm{REM} / \mathrm{AHI}-\mathrm{NREM} \geq 2$, $24.4 \%$ using a total $\mathrm{AHI} \geq 5$, AHI-REM/AHI-NREM $\geq 2$, and $\mathrm{AHI}-\mathrm{NREM}<15$, and $13.5 \%$ using a total $\mathrm{AHI} \geq 5$, AHI$\mathrm{REM} / \mathrm{AHI}-\mathrm{NREM} \geq 2$, AHI-NREM $<8$, and at least $10.5 \mathrm{~min}$ of REM sleep. ${ }^{8}$ Similar findings were reported by Mano et al in a recent Japanese study. ${ }^{21}$

Moreover, the diagnostic definition used of hypopnea affects the reported prevalence of REM-related OSA. Using the 2012 " $3 \%$ desaturation and/or arousal" detects a lower percentage of REM-related OSA cases compared with the 2007 " $4 \%$ desaturation criteria", as it may decrease the REM-AHI/NREM-AHI ratio. ${ }^{23}$ In the current paper, we used conservative criteria that applied the REMAHI/NREM-AHI ratio, the 2012 diagnostic criteria of hypopnea, NREM-AHI $<15$, and minimum sleep duration. Nevertheless, despite variations in the used definitions of REM-related OSA, the current study and previous community-based and clinic population studies demonstrated that REM-related OSA is prevalent.

In the current study, the prevalence of REMpredominant OSA in women was double that in men, and female sex was an independent correlate of REMpredominant OSA. This finding is consistent with previous studies that assessed gender differences in OSA and reported a clustering of respiratory events during REM sleep in women, compared with men who had mainly NREM obstructive events. ${ }^{9,21,24,25}$ Additionally, patients with REM-predominant OSA were younger, which raises the question of whether age or menopausal status is the culprit in women. Therefore, we assessed the independent correlates of REM-predominant OSA among women. Menopausal status and age were correlates in the univariable analysis model; however, in the multiple logistic regression model, only age remained as an independent correlate of REM-predominant OSA. A recent study demonstrated that REM-related OSA is more common in women, irrespective of menopausal status (crudely defined by age $>50$ years $)^{21}$ This is consistent with our findings, which demonstrated that age and female sex are independent correlates, but not menopausal status.

It has also been proposed that female hormones (estrogen and progesterone) may protect women against OSA by increasing the tonicity of the upper airway muscles and stimulating ventilation. ${ }^{26}$ However, it is possible that the hormonal protective effects may not protect women against airway closure during REM sleep. This could be related to the atonia that accompanies REM sleep, which requires more research to elucidate this relationship. In addition, sex affects the pharyngeal area; ${ }^{26}$ therefore, it is possible that the pharyngeal wall in women is more prone to collapse during REM sleep due to its smaller size. Moreover, as obesity was more prevalent among patients with REM-predominant OSA, it is possible that obesity may have a higher impact on the upper airway of women during REM sleep due to its smaller size. 9

In the current study, the ESS was lower in REMpredominant OSA than in NSS-OSA and was independently associated with REM-predominant OSA after adjusting for demographics, BMI, and PSG parameters. Although sleepiness usually correlates with OSA severity, ${ }^{27}$ studies that assessed patients with REM-related OSA reported less association with daytime sleepiness. ${ }^{27-29}$ However, there is no consistency on data related to sleepiness in REM-related OSA; a clinic-based study of 1821 subjects who underwent both PSG and multiple sleep latency reported that after adjusting for age, sex, BMI, and the duration of NREM and REM sleep, the REM-related OSA severity was not associated with daytime sleepiness. ${ }^{29}$ Similarly, in the Sleep Heart Health Study, REM-related OSA was not associated 
Table 6 The Independent Correlates of REM-Predominant OSA According to the Univariable and Multiple Logistic Regression in Men and Women

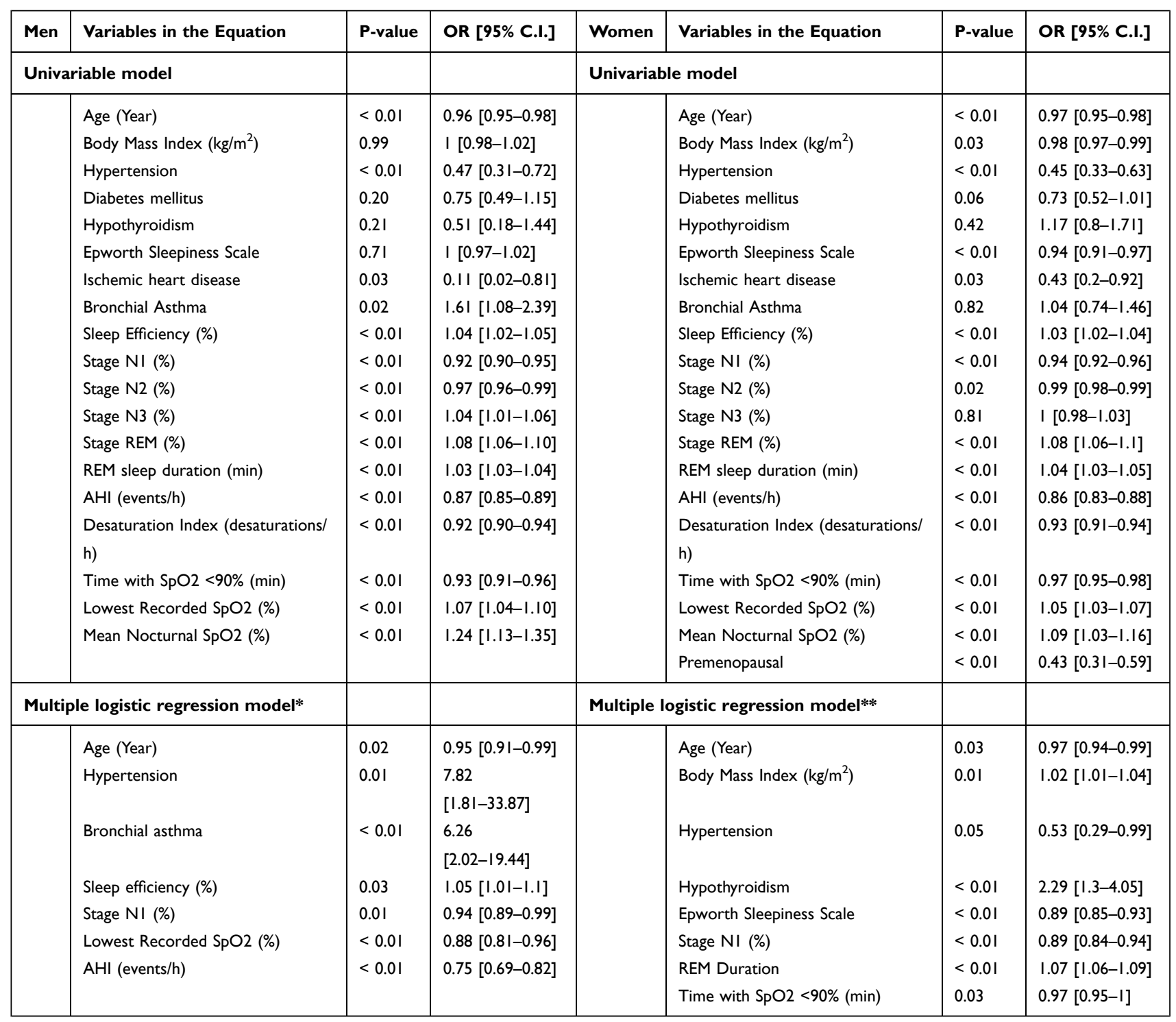

Notes: *Multicollinearity: None. Overall accuracy: $97.7 \%$, Sensitivity: $85.1 \%$, Specificity: $98.8 \%$, Area under the Curve (ROC): $99.7 \%$, Omnibus Tests of Model: p<0.0I, Hosmer-Lemeshow goodness of fit: $p=1.00$, Nagelkerke R Square: $89.6 \%$. **Multicollinearity: None. Overall accuracy: $83.7 \%$, Sensitivity: $55.2 \%$, Specificity: $92.4 \%$, Area under the Curve (ROC): $91.4 \%$, Omnibus Tests of Model: $p<0.01$, Hosmer-Lemeshow goodness of fit: $p=0.02$, Nagelkerke R Square: $55.5 \%$.

Abbreviations: AHI, apnea-hypopnea index; REM, rapid eye movement; OSA, obstructive sleep apnea; SpO2, arterial oxygen saturation; h, hour; min, minutes; kg/m², kilogram per squared meter.

with daytime sleepiness measured by the ESS after adjusting for demographics, BMI, and NREM-AHI. ${ }^{28}$

Insomnia was also more common in patients with REM-predominant OSA than in patients with NSS-OSA. A recent study reported the Pittsburgh Sleep Quality Index (PSQI) scores in 1736 patients with OSA; REM-related OSA was significantly associated with an increased PSQI score. ${ }^{30}$ Snoring was less common among patients with REM-predominant OSA (OR: 0.67, CI: 0.512-0.896). This could be related to the intensity of snoring during different sleep stages. It has been shown that the intensity of snoring was greater during NREM sleep, particularly in stage N3, and less pronounced in REM sleep. ${ }^{31,32}$ Additionally, REM-predominant OSA was more prevalent among women, and it is known that snoring intensity $(\mathrm{dB})$ is commonly higher in men compared to women, ${ }^{33}$ and, further, that it correlates with AHI, which is lower during REM-predominant OSA. ${ }^{31,33}$

Interestingly, hypertension and a history of ischemic heart disease were more common in NSS-OSA. This can be partially explained by the fact that the NSS-OSA group was older and had a higher BMI. Therefore, the current 
Table 7 A Summary of the Studies That Assessed the Prevalence of REM-OSA in the Sleep Laboratory Setting and the Community

\begin{tabular}{|c|c|c|c|c|}
\hline Authors & Study design & Age \& BMI & REM-OSA Definition & Findings \\
\hline \multicolumn{5}{|c|}{ Clinic-based studies } \\
\hline $\begin{array}{l}\text { O'Connor } \\
\text { et al } 2000^{18}\end{array}$ & $\begin{array}{l}\text { A retrospective study of } 830 \\
\text { patients with OSA diagnosed } \\
\text { by overnight PSG }\end{array}$ & $\begin{array}{l}\text { Age: } \\
\text { Women } \\
50.8 \pm 0.9 \\
\text { Men: } 48.6 \\
\pm 0.5 \\
\text { BMI: } 35.1 \\
\pm 0.6 \\
32.1 \pm 0.3\end{array}$ & $\begin{array}{l}\text { Total AHI between } 5 / \mathrm{h} \text { and } 25 / \mathrm{h} \text {, an } \\
\text { NREM AHI }<\text { I5/h, and REM AHI/ } \\
\text { NREM AHI }>2\end{array}$ & $\begin{array}{l}\text { REM-OSA was diagnosed in } 24 \% \text { of } 632 \\
\text { men and } 62 \% \text { of } 206 \text { women } \\
\text { with PSG-identified OSA }\end{array}$ \\
\hline $\begin{array}{l}\text { Koo et al } \\
2008^{17}\end{array}$ & $\begin{array}{l}2486 \text { patients referred to } \\
\text { a sleep laboratory }\end{array}$ & $\begin{array}{l}\text { Age: } 50.8 \pm \\
0.3 \text { BMI: } \\
30.8 \pm 0.1\end{array}$ & $\begin{array}{l}\text { NREM-AHI } \leq I 5 / h \text { and REM-AHI } \\
\text { /NREM-AHI ratio } \geq 2\end{array}$ & $\begin{array}{l}21 \% \text { of men and } 40.8 \% \text { of women had } \\
\text { REM-OSA } \\
\text { Female sex remained an independent } \\
\text { predictor of REM-OSA (OR, 3.0). REM- } \\
\text { OSA prevalence decreased with } \\
\text { increasing age in both sexes }\end{array}$ \\
\hline $\begin{array}{l}\text { Resta et al } \\
2005^{19}\end{array}$ & $\begin{array}{l}\text { A cross-sectional study of } 45 \\
\text { obese subjects, } 20 \text { females and } \\
25 \text { males }\end{array}$ & $\begin{array}{l}\text { Age: } \\
\text { Men } 45 \pm 10 \\
\text { Women } 43 \\
\pm 13.5 \\
\text { BMI: } \\
\text { Men: } 40 \pm 10 \\
\text { Women:39.7 } \\
\pm 7\end{array}$ & $\begin{array}{l}\text { Total AHI between } 5 / h \text { and } 25 / h \text {, } \\
\text { an NREM-AHI }<15 / h \text {, and REM-AHI } \\
\text { /NREM AHI }>2\end{array}$ & $\begin{array}{l}\text { REM-OSA was diagnosed in } 4 \% \text { of men } \\
\text { and } 35 \% \text { of women }\end{array}$ \\
\hline $\begin{array}{l}\text { Haba-Rubio } \\
\text { et al } 2005^{4}\end{array}$ & $\begin{array}{l}\text { A cross-sectional study of } 415 \\
\text { patients with OSA }\end{array}$ & $\begin{array}{l}\text { Age; } 53 \\
\pm 11.6\end{array}$ & An AHI-REM/AHI-NREM ratio $>2$. & $\begin{array}{l}\text { REM-OSA was diagnosed in } 36 \% \text { of } \\
\text { OSA patients }\end{array}$ \\
\hline $\begin{array}{l}\text { Koo et al } \\
2008^{9}\end{array}$ & $\begin{array}{l}\text { A retrospective study of } 1540 \\
\text { OSA patients. }\end{array}$ & $\begin{array}{l}\text { Age: } 50 \pm 0.8 \\
\text { BMI: } 35.7 \\
\pm 0.7\end{array}$ & $\begin{array}{l}\text { NREM-AHI }<15 \text {, and REM-AHI } \\
\text { /NREM-AHI }>2\end{array}$ & $\begin{array}{l}\text { REM-OSA was diagnosed in } 14.4 \% \text { of } \\
\text { patients }\end{array}$ \\
\hline $\begin{array}{l}\text { Conwell } \\
\text { et al } 2012^{8}\end{array}$ & $\begin{array}{l}\text { A cross-section study of } \\
\text { patients referred to a sleep } \\
\text { laboratory }(n=931)\end{array}$ & $\begin{array}{l}\text { Age: } 45 \pm 15 \\
\text { BMI: } 36 \pm 9\end{array}$ & $\begin{array}{l}\text { Three definitions: } \\
\text { I. Overall } A H I \geq 5 \text { and AHI-REM/AHI- } \\
\text { NREM } \geq 2 \\
\text { 2. Overall } A H I \geq 5 \text {, AHI-REM/AHI- } \\
\text { NREM } \geq 2 \text {, and AHI-NREM }<15 \\
\text { 3. Overall AHI } \geq 5 \text {, AHI-REM/AHI- } \\
\text { NREM } \geq 2 \text {, AHI-NREM }<8 \text {, and at least } \\
\text { I0.5 min of REM sleep duration }\end{array}$ & $\begin{array}{l}\text { I. REM-OSA was diagnosed in } 37 \% \\
\text { when only REM-AHI/NREM-AHI } 2 \text { was } \\
\text { used, } \\
\text { 2. } 24.4 \% \text { when the criterion of NREM } \\
\text { AHI }<15 / h \text { was added, } \\
\text { 3. } 13.5 \% \text { when the criterion of } \\
\text { NREM AHI }<8 / \mathrm{h} \text { and at least at least } \\
\text { I0.5 min of REM sleep duration were } \\
\text { added }\end{array}$ \\
\hline $\begin{array}{l}\text { Mano et al } \\
2019^{21}\end{array}$ & $\begin{array}{l}\text { A total of } 3234 \text { Japanese } \\
\text { patients with OSA }\end{array}$ & $\begin{array}{l}\text { Age: } \\
\text { Women: } \\
57.1 \pm 13.8 \\
\text { Men: } 51.7 \pm \\
\text { I3.5 } \\
\text { BMI: } \\
\text { Women: } \\
27.2 \pm 7.9 \\
\text { Men: } 26.9 \pm \\
5.0\end{array}$ & $\begin{array}{l}\text { Three definitions: } \\
\text { I. Total AHI>5 and REM-AHI } \\
\text { /NREM-AHI>2; } \\
\text { 2. AHI>5 and REM-AHI/NREM- } \\
A H I>2 \text {, and NREM-AHI<I5; } \\
\text { 3. AHI>5 and REM-AHI/NREM- } \\
A H I>2 \text {, and NREM-AHI<8, and } \\
\text { REM sleep > } 10.5 \mathrm{~min}\end{array}$ & $\begin{array}{l}\text { The prevalence in women and men } \\
\text { according to diagnostic criteria \#I, \#2, } \\
\text { and \#3 were } 47.7 \text { vs. } 19 \%, 37.5 \% \text { vs. } \\
14.5 \% \text {, and } 26.3 \% \text { vs. } 8.7 \% \text {, respectively. }\end{array}$ \\
\hline
\end{tabular}


Table 7 (Continued).

\begin{tabular}{|c|c|c|c|c|}
\hline Authors & Study design & Age \& BMI & REM-OSA Definition & Findings \\
\hline $\begin{array}{l}\text { Al Oweidat } \\
\text { et al. } 2018^{20}\end{array}$ & $\begin{array}{l}\text { A cross-sectional study of } 478 \\
\text { patients }\end{array}$ & $\begin{array}{l}\text { Age: } 55.3 \\
\pm I 2.6 \\
\text { BMI: } 36.6 \\
\pm 7.6\end{array}$ & $\begin{array}{l}\text { Two criteria } \\
\text { I. AHI-REM/AHI-NREM at least } 2 \text {. } \\
\text { 2. AHI-REM } \geq 5 \text { and AHI-NREM }<5 \text {, } \\
\text { with a total REM sleep of } \geq 30 \\
\text { min. }\end{array}$ & $\begin{array}{l}\text { REM-OSA prevalence: } \\
\text { Criteria \#I: } 18 \% \\
\text { Criteria \#2: } 2.7 \%\end{array}$ \\
\hline $\begin{array}{l}\text { Current } \\
\text { study } \\
\text { Bahammam } \\
\text { et al } 2020\end{array}$ & $\begin{array}{l}\text { Prospectively collected data } \\
\text { on } 2169 \text { OSA patients }\end{array}$ & & $\begin{array}{l}\text { Obstructive } A H I \text { of } \geq 5 \text {, with REM- } \\
A H I / N R E M-A H I \text { of }>2 \text {, an NREM- } \\
A H I \text { of }<15 \text {, and a minimum of } 15 \\
\text { min of REM sleep }\end{array}$ & $\begin{array}{l}\text { REM-predominant OSA was diagnosed } \\
\text { in } 17 \% \text {. The prevalence of REM- } \\
\text { predominant OSA in women and men } \\
\text { was } 25 \% \text { and I } 2 \% \text {, respectively }\end{array}$ \\
\hline \multicolumn{5}{|c|}{ Community-based epidemiological data } \\
\hline $\begin{array}{l}\text { Mokhlesi } \\
\text { et al } 2014^{7}\end{array}$ & $\begin{array}{l}\text { The longitudinal community- } \\
\text { based Wisconsin Sleep Cohort } \\
\text { Study ( } n=4385 \text { sleep studies } \\
\text { on } 145 \text { I individuals) }\end{array}$ & $\begin{array}{l}\text { Age: } 54 \pm 10 \\
\text { BMI: } 30 \pm 6\end{array}$ & $\begin{array}{l}30 \text { min of REM and an NREM AHI } \\
(4 \%) \leq 5 \text { events/h }\end{array}$ & $\begin{array}{l}\text { I } 2 \% \text { demonstrated a REM-AHI } \geq \\
\text { I } 5 \text { events } / \mathrm{h} \\
\text { Among studies where the total } \\
\text { AHI was }<15 \text { events } / \mathrm{h}, 22 \% \\
\text { demonstrated a REM-AHI } \geq 15 \text { events } / \mathrm{h} \text {. }\end{array}$ \\
\hline $\begin{array}{l}\text { Aurora et al } \\
2018^{15}\end{array}$ & $\begin{array}{l}\text { The Sleep Heart Health Study } \\
(n=3265)\end{array}$ & $\begin{array}{l}\text { Age: } 62 \\
\pm 10.7 \\
\text { BMI: } 27.8 \pm 5\end{array}$ & $\begin{array}{l}\text { At least } 30 \text { min of REM sleep and an } \\
\text { NREM AHI } 4 \%<5 \text { events } / \mathrm{h}\end{array}$ & $\begin{array}{l}27.7 \% \text { had a REM-AHI of } 5.0-14.9 \\
\text { events/h, I3\% had a REM-AHI of } \\
\text { I5.0-29.9 events/ } \\
\text { h, and } 5.5 \% \text { had a REM-AHI > } 30 \text { events/ } \\
\text { h }\end{array}$ \\
\hline $\begin{array}{l}\text { Khan et al } \\
2013^{16}\end{array}$ & $\begin{array}{l}\text { A cross-sectional analysis of } \\
2765 \text { Older Men, the (MrOS } \\
\text { Sleep) Study }\end{array}$ & $\begin{array}{l}\text { Age: } 76 \\
\text { BMI: } 25-29\end{array}$ & $\begin{array}{l}\text { OSA was defined as } A H I \geq I 5 \text { during } \\
\text { the entire sleep period. REM- } \\
\text { predominant OSA was defined as } \\
A H I<15 \text {, but REM-AHI } \geq 5\end{array}$ & $\begin{array}{l}\text { REM-predominant OSA (AHI-REM } \geq 5 \text { ) } \\
\text { was } 42.8 \% \text { if OSA was defined as } A H I \geq \\
I 5 \text { and } I 4.4 \% \text { if OSA was defined as } A H I \\
\geq 5 \text {. }\end{array}$ \\
\hline
\end{tabular}

Abbreviations: REM, rapid eye movement; NREM, non-rapid eye movement; BMI, body mass index; AHI, apnea-hypopnea index.

results should not be mistakenly interpreted that REMpredominant OSA has a lower risk of cardiac morbidity, as community-based studies have suggested that REMrelated OSA is significantly associated with hypertension, independent of NREM-OSA.,34,35 Moreover, the NSSOSA group had a high REM-AHI of $51.3 \pm 36.7 / \mathrm{h}$; however, the ratio of REM-AHI-NREN-AHI was less than 2 .

Another interesting finding is the fact that bronchial asthma was an independent correlate of REM-predominant OSA. Recent data have indicated a strong link between bronchial asthma and OSA, while showing a bidirectional relationship between the two disorders. ${ }^{36}$ An earlier study has demonstrated that adults with stable asthma have a cluster of irregular breathing and hypoxemia during REM sleep. ${ }^{37}$ Studies in mice revealed that REM sleep is associated with increasing cholinergic outflow and weardown of noradrenergic signals in the brainstem, which are relevant controllers of the lower airways' caliber and reactivity. ${ }^{38}$ In an earlier study, Shapiro et al assessed the relationship between bronchoconstriction and sleep stages. ${ }^{39}$ The investigators performed forced expiratory maneuvers, directly after being awakened from REM or NREM sleep, on eight patients with asthma and eight controls. After patients with asthma were awakened from REM sleep, the forced expiratory volume in one second (FEV1) and peak expiratory flow (PEF) rate were significantly lower than after they had been awakened from NREM sleep. ${ }^{39}$ A subsequent multiple logistic regression analysis was performed to account for possible temporal effects from those related to the stage of sleep, demonstrating that the overnight decrement in FEV1 and PEF rate were significantly related to REM sleep. ${ }^{39}$ Recent studies in children revealed that REM-related OSA is significantly increased in children with OSA and comorbid asthma compared to children with OSA alone and that the association between asthma and REM-related OSA was independent of asthma control, BMI, age, and sex. ${ }^{40,41}$ Therefore, it seems that there is an association between asthma and REM-related 
OSA. Further research is warranted to explain the REM sleep neurobiological mechanisms that potentially influence OSA expression in patients with asthma.

The current study has strengths and limitations. Strengths include having clear inclusion and exclusion criteria and using strict criteria to define REMpredominant OSA, including a cut-off point for NREMAHI, REM-AHI/NREM-AHI ratio, and minimum duration for REM sleep. The large sample size represents another strength. Additionally, this is the first study to assess the association between REM-related OSA and menopausal status using a clear definition of menopause.

Limitation includes the fact that the study was conducted in a single center. Additionally, being a clinic-based study means that there could be a selection bias, as symptomatic patients are usually referred to the clinic.

\section{Conclusion}

The current study demonstrated that REM-predominant OSA, using strict criteria to define it, is prevalent among clinic-based patients with OSA. Younger age and female sex were among the independent associations of REMpredominant OSA. Among women, a younger age but not menopausal status was an independent correlate of REM-predominant OSA. Interestingly, bronchial asthma was independently associated with REM-predominant OSA, supporting previous experimental data suggesting an association between asthma and REM-related OSA.

\section{Abbreviations}

REM, rapid eye movement; NSS, non-stage-specific; OSA, obstructive sleep apnea; NREM, non-rapid eye movement; PSG, polysomnography; MSLT, multiple sleep latency test; BMI, body mass index; ESS, Epworth sleepiness scale; AHI, apnea-hypopnea index; SPO2, oxygen saturation; SD, standard deviation; AASM, American Academy of Sleep Medicine; dB, decibel; FEV1, forced expiratory volume in one second; PEF, peak expiratory flow.

\section{Data Sharing Statement}

Data are available on request; however, an institutional approval is needed before sharing.

\section{Ethics Approval and Informed Consent}

The study was approved by the Institutional Review Board of the Health Sciences Colleges Research on Human
Subjects at King Saud University (E-19-4169). All participants signed a written informed consent form. This study was conducted in accordance with the Declaration of Helsinki.

\section{Acknowledgments}

This study was funded by a grant from the Strategic Technologies Program of the National Plan for Sciences and Technology and Innovation in the Kingdom of Saudi Arabia (08-MED511-02). The study sponsor played no role in the study design, the collection, analysis or interpretation of data, writing the manuscript, or the decision to submit the manuscript.

\section{Author Contributions}

All authors contributed to data analysis, drafting or revising the article, have agreed on the journal (Nature and Science of Sleep) to which the article will be submitted, gave final approval of the version to be published, and agree to be accountable for all aspects of the work.

\section{Disclosure}

All authors declare that they have no proprietary, financial, professional, or other personal interest of any nature in any product, service, and/or company that could be construed or considered to be a potential conflict of interest that might have influenced the views expressed in this manuscript.

\section{References}

1. Senaratna CV, Perret JL, Lodge CJ, et al. Prevalence of obstructive sleep apnea in the general population: a systematic review. Sleep Med Rev. 2017;34:70-81.

2. Benjafield AV, Ayas NT, Eastwood PR, et al. Estimation of the global prevalence and burden of obstructive sleep apnoea: a literature-based analysis. Lancet Respir Med. 2019;7(8):687-698. doi:10.1016/S22132600(19)30198-5

3. Fenik VB, Davies RO, Kubin L. REM sleep-like atonia of hypoglossal (XII) motoneurons is caused by loss of noradrenergic and serotonergic inputs. Am J Respir Crit Care Med. 2005;172(10):1322-1330. doi:10.1164/rccm.200412-1750OC

4. Haba-Rubio J, Janssens JP, Rochat T, Sforza E. Rapid eye movement-related disordered breathing: clinical and polysomnographic features. Chest. 2005;128(5):3350-3357. doi:10.1378/ chest.128.5.3350

5. Almeneessier AS, Nashwan SZ, Olaish A, Al Anbi E, BaHammam AS. Long-term adherence to continuous positive airway pressure in patients with REM-only obstructive sleep apnea: a prospective cohort study. J Thorac Dis. 2017;9(10):3755-3765. doi:10.21037/jtd.2017.09.57

6. Almeneessier AS, Alshahrani M, Aleissi S, Hammad OS, Olaish AH, BaHammam AS. Comparison between blood pressure during obstructive respiratory events in REM and NREM sleep using pulse transit time. Sci Rep. 2020;10(1):3342. doi:10.1038/s41598-020-60281-2 
7. Mokhlesi B, Finn LA, Hagen EW, et al. Obstructive sleep apnea during REM sleep and hypertension. Results of the wisconsin sleep cohort. Am J Respir Crit Care Med. 2014;190(10):1158-1167. doi:10.1164/rccm.201406-1136OC

8. Conwell W, Patel B, Doeing D, et al. Prevalence, clinical features, and CPAP adherence in REM-related sleep-disordered breathing: a cross-sectional analysis of a large clinical population. Sleep Breath. 2012;16(2):519-526. doi:10.1007/s11325-011-0537-6

9. Koo BB, Dostal J, Ioachimescu O, Budur K. The effects of gender and age on REM-related sleep-disordered breathing. Sleep Breath. 2008;12(3):259-264. doi:10.1007/s11325-007-0161-7

10. Czeisler CA, Zimmerman JC, Ronda JM, Moore-Ede MC, Weitzman ED. Timing of REM sleep is coupled to the circadian rhythm of body temperature in man. Sleep. 1980;2(3):329-346. doi:10.1093/sleep/2.3.329

11. Dalal PK, Agarwal M. Postmenopausal syndrome. Indian $J$ Psychiatry. 2015;57(Suppl 2):S222-S232. doi:10.4103/00195545.161483

12. BaHammam AS, Pandi-Perumal SR, Piper A, et al. Gender differences in patients with obesity hypoventilation syndrome. J Sleep Res. 2016;25(4):445-453. doi:10.1111/jsr. 12400

13. Johns MW. Reliability and factor analysis of the epworth sleepiness scale. Sleep. 1992;15(4):376-381. doi:10.1093/sleep/15.4.376

14. Berry RB, Brooks R, Gamaldo C, et al. AASM scoring manual updates for 2017 (version 2.4). J Clin Sleep Med. 2017;13 (5):665-666. doi:10.5664/jcsm.6576

15. Aurora RN, Crainiceanu C, Gottlieb DJ, Kim JS, Punjabi NM. Obstructive sleep apnea during REM sleep and cardiovascular disease. Am J Respir Crit Care Med. 2018;197(5):653-660. doi:10.1164/rccm.201706-1112OC

16. Khan A, Harrison SL, Kezirian EJ, et al. Obstructive sleep apnea during rapid eye movement sleep, daytime sleepiness, and quality of life in older men in Osteoporotic Fractures in Men (MrOS) Sleep Study. J Clin Sleep Med. 2013;9(3):191-198.

17. Koo BB, Patel SR, Strohl K, Hoffstein V. Rapid eye movement-related sleep-disordered breathing: influence of age and gender. Chest. 2008;134(6):1156-1161. doi:10.1378/chest.08-1311

18. O'Connor C, Thornley KS, Hanly PJ. Gender differences in the polysomnographic features of obstructive sleep apnea. Am J Respir Crit Care Med. 2000;161(5):1465-1472.

19. Resta O, Carpanano GE, Lacedonia D, et al. Gender difference in sleep profile of severely obese patients with obstructive sleep apnea (OSA). Respir Med. 2005;99(1):91-96. doi:10.1016/j.rmed.2004. 05.014

20. Al Oweidat K, AlRyalat SA, Al-Essa M, Obeidat N. Comparing REM- and NREM-related obstructive sleep apnea in Jordan: a Cross-Sectional Study. Can Respir J. 2018;2018:9270329. doi:10.1155/2018/9270329

21. Mano M, Hoshino T, Sasanabe R, et al. Impact of gender and age on rapid eye movement-related obstructive sleep apnea: a Clinical Study of 3234 Japanese OSA patients. Int J Environ Res Public Health. 2019;16(6):1068. doi:10.3390/ijerph16061068

22. Mokhlesi B, Punjabi NM. "REM-related" obstructive sleep apnea: an epiphenomenon or a clinically important entity? Sleep. 2012;35 (1):5-7. doi:10.5665/sleep.1570

23. Duce B, Kulkas A, Langton C, Toyras J, Hukins C. The prevalence of REM-related obstructive sleep apnoea is reduced by the AASM 2012 hypopnoea criteria. Sleep Breath. 2018;22(1):57-64. doi:10.1007/ s11325-017-1526-1
24. Lin CM, Davidson TM, Ancoli-Israel S. Gender differences in obstructive sleep apnea and treatment implications. Sleep Med Rev. 2008;12(6):481-496. doi:10.1016/j.smrv.2007.11.003

25. Alotair H, Bahammam A. Gender differences in Saudi patients with obstructive sleep apnea. Sleep Breath. 2008;12(4):323-329. doi:10. 1007/s11325-008-0184-8

26. LoMauro A, Aliverti A. Sex differences in respiratory function. Breathe (Sheff). 2018;14(2):131-140. doi:10.1183/20734735.000318

27. Varga AW, Mokhlesi B. REM obstructive sleep apnea: risk for adverse health outcomes and novel treatments. Sleep Breath. 2019;23(2):413-423. doi:10.1007/s11325-018-1727-2

28. Chami HA, Baldwin CM, Silverman A, et al. Sleepiness, quality of life, and sleep maintenance in REM versus non-REM sleep-disordered breathing. Am J Respir Crit Care Med. 2010;181 (9):997-1002. doi:10.1164/rccm.200908-1304OC

29. Punjabi NM, Bandeen-Roche K, Marx JJ, Neubauer DN, Smith PL, Schwartz AR. The association between daytime sleepiness and sleep-disordered breathing in NREM and REM sleep. Sleep. 2002;25(3):307-314.

30. Hoshino T, Sasanabe R, Murotani K, et al. Insomnia as a symptom of rapid eye movement-related obstructive sleep apnea. J Clin Med. 2020;9(6):1821.

31. Levartovsky A, Dafna E, Zigel Y, Tarasiuk A. Breathing and snoring sound characteristics during sleep in adults. $J$ Clin Sleep Med. 2016;12(3):375-384. doi:10.5664/jcsm.5588

32. Perez-Padilla JR, West P, Kryger M. Snoring in normal young adults: prevalence in sleep stages and associated changes in oxygen saturation, heart rate, and breathing pattern. Sleep. 1987;10(3):249-253. doi:10.1093/sleep/10.3.249

33. Pevernagie D, Aarts RM, De Meyer M. The acoustics of snoring. Sleep Med Rev. 2010;14(2):131-144. doi:10.1016/j.smrv.2009.06.002

34. Acosta-Castro P, Hirotsu C, Marti-Soler H, et al. REM-associated sleep apnoea: prevalence and clinical significance in the HypnoLaus cohort. Eur Respir J. 2018;52(2):1702484. doi:10.1183/ 13993003.02484-2017

35. Appleton SL, Vakulin A, Martin SA, et al. Hypertension is associated with undiagnosed OSA during rapid eye movement sleep. Chest. 2016;150(3):495-505. doi:10.1016/j.chest.2016.03.010

36. Kong DL, Qin Z, Shen H, Jin HY, Wang W, Wang ZF. Association of obstructive sleep apnea with asthma: a meta-analysis. Sci Rep. 2017;7 (1):4088. doi:10.1038/s41598-017-04446-6

37. Catterall JR, Douglas NJ, Calverley PM, et al. Irregular breathing and hypoxaemia during sleep in chronic stable asthma. Lancet. 1982;1 (8267):301-304. doi:10.1016/S0140-6736(82)91567-7

38. Haxhiu MA, Kc P, Moore CT, et al. Brain stem excitatory and inhibitory signaling pathways regulating bronchoconstrictive responses. J Appl Physiol. 2005;98(6):1961-1982. doi:10.1152/ japplphysiol.01340.2004

39. Shapiro CM, Catterall JR, Montgomery I, Raab GM, Douglas NJ. Do asthmatics suffer bronchoconstriction during rapid eye movement sleep? $B r \quad$ Med J. 1986;292(6529):1161-1164. doi:10.1136/ bmj.292.6529.1161

40. Gutierrez MJ, Zhu J, Rodriguez-Martinez CE, Nino CL, Nino G. Nocturnal phenotypical features of obstructive sleep apnea (OSA) in asthmatic children. Pediatr Pulmonol. 2013;48(6):592-600.

41. Perez GF, Gutierrez MJ, Huseni S, et al. Oximetry signal processing identifies REM sleep-related vulnerability trait in asthmatic children. Sleep Disord. 2013;2013:406157. doi:10.1155/2013/406157 


\section{Publish your work in this journal}

Nature and Science of Sleep is an international, peer-reviewed, open access journal covering all aspects of sleep science and sleep medicine, including the neurophysiology and functions of sleep, the genetics of sleep, sleep and society, biological rhythms, dreaming, sleep disorders and therapy, and strategies to optimize healthy sleep.
The manuscript management system is completely online and includes a very quick and fair peer-review system, which is all easy to use. Visit http://www.dovepress.com/testimonials.php to read real quotes from published authors. 